\title{
Medición de la relevancia y evolución de la bioeconomía en España
}

\section{Measuring the Relevance and the Evolution of the Bioeconomy in Spain}

\author{
Encarna Esteban \\ Alexia Sanz-Hernández \\ Universidad de Zaragoza
}

\section{Resumen}

Durante las últimas décadas la bioeconomía se ha impuesto como una opción para garantizar el crecimiento económico y la sostenibilidad de los recursos naturales. Muchos países a nivel internacional han incluido a la bioeconomía dentro de su agenda política como una alternativa de desarrollo económico. El objetivo de este estudio es analizar la relevancia y evolución de la bioeconomía en España a través del análisis de distintos indicadores macroeconómicos. España presentó su estrategia nacional de bioeconomía en 2016 y, en los últimos años, los datos reflejan un incremento en la contribución al producto interior bruto, así como en la generación de puestos de empleo en los sectores asociados a la bioeconomía. Además, los resultados muestran el notable esfuerzo que se está realizando en el contexto de la investigación y la innovación en este sector. Sin embargo, todavía la distancia con respecto a otros países europeos es notable y existen fuertes disparidades territoriales y numerosos retos nacionales en la transición hacia una economía biobasada sostenible.

Palabras clave: bioeconomía, valor añadido, empleo, $I+D+i$, estrategia de bioeconomía, biotecnología.

Clasificación JEL: O13, Q01, Q20.

\begin{abstract}
During the last decades, the bioeconomy has established itself as an option for ensuring economic growth and guaranteeing the sustainability of natural resources. Several countries have included the bioeconomy in their political agendas as an alternative for economic development. This paper aims to analyze the relevance and evolution of the bioeconomy in Spain based on the analysis of different macroeconomic variables and indicators. Spain developed its bioeconomy strategy in 2016 and the analysis of the data shows how this economy has advanced in the contribution of the bioeconomy to the Gross Domestic Product and in the generation of jobs related to this sector. Furthermore, important efforts have been made in the context of research and development. Despite the growth of the bioeconomy in Spain, this country is still far from many of the European states and a lot of work is still pending to be done.
\end{abstract}

Keywords: bioeconomy, value added, employment, research and innovation (R\&D), bioeconomy strategies, biotechnology. 


\section{Introducción}

El crecimiento de la población mundial y el actual modelo de desarrollo económico ha generado una fuerte presión sobre los recursos naturales que, en muchos casos, se encuentran al límite de su capacidad. La preocupación por el medioambiente ha llevado a los distintos países a la elaboración de marcos normativos para la regulación, control y protección de los recursos naturales (O’Brien, 2011). Sin embargo, a pesar de los numerosos esfuerzos nacionales e internacionales, la protección del medioambiente sigue siendo un tema pendiente y clave en todas las agendas políticas de países e instituciones. En este contexto, la bioeconomía se está imponiendo como una opción alternativa para preservar los recursos naturales y garantizar el desarrollo social y el crecimiento económico (D'Amato et al., 2017; Bugge et al., 2016; Aquilani et al., 2018). El modelo bioeconómico supone el mantenimiento y la sostenibilidad de los sistemas productivos en su triple dimensionalidad: económica, social y ambiental (Comisión Europea, 2018).

El concepto de bioeconomía fue acuñado en los años setenta por Georgescu-Roegen, remarcando la necesidad de incorporar los procesos biológicos y biofísicos dentro de la teoría económica (Georgescu-Roegen, 1977). Según este autor, la economía debía incorporar y fusionar en sus teorías procesos de la naturaleza. A lo largo de los años este concepto se ha ido reformulando y actualmente la bioeconomía puede definirse como una propuesta económica en la que los procesos productivos de determinados sectores tales como el energético, químico, forestal, agrícola o agroalimentario, entre otros, están directamente ligados al uso de los recursos naturales (McCormick \& Kautto, 2013). La bioeconomía se refiere por tanto al conjunto de actividades relacionadas con la investigación, desarrollo, producción y aprovechamiento de productos y/o procesos biológicos y biotecnológicos para garantizar un desarrollo económico sostenible (OCDE, 2019; Ingrao et al., 2018; Sanz-Hernández, Esteban et al., 2019).

Durante las últimas décadas se han desplegado numerosas estrategias para potenciar la implementación de un modelo bioeconómico (OCDE, 2004). Actualmente, más de 50 países en todo el mundo, y 30 en el contexto europeo, han implementado o están en proceso de elaboración de estrategias nacionales de bioeconomía o estrategias relacionadas con la bioeconomía (OCDE, 2018). España es actualmente uno de los países que ha mostrado un mayor interés por la bioeconomía al diseñar una estrategia nacional (Ministerio de Economía y Competitividad, 2018). Además, actualmente se encuentra en proceso de debate público para su aprobación la Estrategia Española de Economía Circular, denominada España Circular 2030 (Gobierno de España, 2020), que incorpora unas líneas específicas dedicadas a la bioeconomía circular. Finalmente, a la estrategia bioeconómica nacional que se viene desarrollando desde 2016, se han sumado estrategias regionales en la mayoría de las comunidades autónomas (CC. AA.), ya sea bajo la denominación de bioeconomía o bien bajo la de economía circular donde se incorpora a la bioeconomía (Sanz-Hernández et al., 2020). 
El objetivo de este trabajo es el de medir la relevancia actual de la bioeconomía y su reciente evolución dentro de la economía española, a través del análisis de una serie de variables macroeconómicas e indicadores considerados relevantes en la literatura más reciente (D’Adamo et al., 2020). Se atiende por un lado a la evolución del valor añadido y la cifra de negocios de los principales sectores asociados con la bioeconomía (a partir de aquí denominados sectores bioeconómicos), especialmente, el sector primario, las bioenergías y las industrias dedicadas a la explotación de recursos naturales. Por otro lado, se ha considerado la evolución del empleo en estos sectores. Un tercer análisis incorpora otro indicador importante como es la inversión tanto pública como privada en I+D+i. Finalmente, se realiza una comparación de la evolución de la bioeconomía en el contexto español con respecto a la Unión Europea (UE).

Los resultados muestran cómo la bioeconomía en España se ha potenciado desde 2008 hasta la actualidad. En 2012, tanto el valor añadido como la cifra de negocio de este sector muestra un crecimiento sostenido que se ha intensificado durante los últimos años. En este sentido, la evolución de España sigue una pauta similar al resto de países de la UE, aunque los resultados comparativos evidencian que el ritmo de crecimiento de la bioeconomía en España está siendo menor que la media de los 27 países que conforman la UE.

Un análisis más detallado de la evolución por sectores bioeconómicos evidencia que durante los últimos años la bioenergía es uno de los sectores con mayor crecimiento en España. Además de la bioenergía, están cobrando importancia otros sectores como la fabricación de productos químicos, farmacéuticos y otros materiales de origen biológico, que en el caso de España parten de niveles más bajos de generación de valor añadido comparado con los principales países de la UE. A pesar de la potenciación de la bioeconomía española, todavía existen numerosas limitaciones y desafíos en España para alcanzar la media europea, tanto en crecimiento de las actividades productivas como en potenciación del empleo en estos sectores. Además, los resultados enseñan que el esfuerzo que se está realizando en la inversión en biotecnologías está todavía muy concentrada en determinadas regiones del país como la Comunidad de Madrid o Cataluña. Por tanto, España tiene que desplegar mayores esfuerzos en potenciar la innovación en los sectores relacionados con la bioeconomía y conseguir un desarrollo mucho más homogéneo y armónico entre los diferentes territorios del país.

El trabajo está organizado de la siguiente manera. En la siguiente sección se analiza la relevancia internacional de la bioeconomía y los principales problemas para su medición. En la tercera sección se analiza la evolución del valor añadido, cifra de negocio y empleo generado de los principales sectores bioeconómicos de España desde 2008. La cuarta sección presenta la situación y evolución de la inversión en $\mathrm{I}+\mathrm{D}+\mathrm{i}$ en los principales sectores bioeconómicos. Finalmente, la última sección presenta una discusión general de los resultados y de la situación de la bioeconomía en España. 


\section{La relevancia de la bioeconomía y su medición}

La bioeconomía se basa en el uso de recursos biológicos y de la biotecnología en los sistemas de producción y, por tanto, está fuertemente ligada a los sectores primario (p. ej., agricultura, pesca y producción forestal) y secundario (industria agroalimentaria y bioquímica, farmacéutica y bioplásticos, entre otros). Igualmente, una línea fundamental dentro de los sectores bioeconómicos es la producción de energía, bioelectricidad y otros combustibles biológicos.

La bioeconomía proyectada como un vínculo entre procesos productivos y medioambiente supone una oportunidad para alcanzar un desarrollo económico sostenible, potenciando la mejora en la calidad de vida y promoviendo el desarrollo de las economías rurales. Durante los últimos años hemos asistido a una fuerte potenciación de la bioeconomía a nivel internacional como base para una transición hacia un modelo de «reindustrialización», centrado tanto en el control y protección del medioambiente como en la garantía del crecimiento económico y la reducción de las desigualdades sociales y territoriales. En este sentido, la Organización para la Cooperación y el Desarrollo Económico (OCDE) ha impulsado un marco estratégico que establece la necesidad de potenciar una sociedad basada en la bioeconomía (OCDE, 2004) a partir de una agenda política que la estimule (OCDE, 2009).

La UE estableció una Directiva para la promoción de la bioeconomía en los países miembros y definió su Estrategia de Bioeconomía en 2012 (Comisión Europea, 2012). La estrategia se actualizó en 2018 con el objetivo de potenciar y acelerar el desarrollo de este modelo y su implantación en los distintos países miembros (Comisión Europea, 2018). El objetivo perseguido era el de potenciar un desarrollo sostenible (Dresner, 2002; Jabareen, 2008), basado en la incorporación en los procesos productivos de materiales de origen biológico, así como de todo el conocimiento y desarrollos en biotecnología en todos los países miembros, con la confianza en que estos puedan fortalecer la innovación y facilitar la inclusión laboral y social de su población. La estrategia de bioeconomía europea es muy ambiciosa y busca la potenciación de aquellos sectores basados en el uso de recursos biológicos y con bajo impacto medioambiental. Pero, además, esta estrategia persigue también el cambio de los actuales modelos de negocios y de los patrones de consumo para garantizar la sostenibilidad medioambiental. La estrategia europea de bioeconomía se asienta en una serie de pilares fundamentales (Bell et al., 2018):

- Recursos: se ha contemplado el incremento en las inversiones en investigación y desarrollo en biotecnologías para la potenciación del uso de biorecursos y la reducción de la huella ecológica.

- Agentes implicados y la sociedad: una parte estratégica esencial es la concienciación de todos los actores acerca de la importancia de un consumo racional de los recursos y la generación de menos residuos. La transición hacia un modelo bioeconómico es impensable sin la implicación social y el cambio en los patrones de consumo que estimulen producciones más limpias y sostenibles. 
- Gobiernos e instituciones: nuevas fórmulas de gobernanza y el apoyo de gobiernos e instituciones son necesarios para la creación de normativas y regulaciones específicas que permitan el desarrollo de este modelo. Asimismo, se hace hincapié en la ineludible colaboración público-privada para incrementar la inversión en sectores estratégicos relacionados con la bioeconomía.

- Mercados: el modelo se asienta en la noción de mejora de la competitividad de la economía europea a través del uso sostenible de los recursos naturales. El nuevo modelo productivo que se propone aspira a compatibilizar la prosperidad económica y el bienestar social reduciendo las desigualdades y garantizando la sostenibilidad ambiental.

En la actualidad, la bioeconomía en el contexto europeo tiene un peso fundamental. Los sectores que engloban la bioeconomía en la $\mathrm{UE}^{1}$ suponen una contribución de 614 billones de euros al total del valor añadido generado en la Unión. Además, estos sectores ocupan a más de 17 millones de trabajadores en toda la zona (Ronzon $e t$ al., 2020)2 . En el Gráfico 1 se recoge la contribución de la bioeconomía por sectores en la Unión Europea, asumiendo la propuesta de agrupación de los 18 microsectores que contempla la clasificación estadística oficial de actividades económicas de la Comunidad Europea (Eurostat, 2008), en los 10 sugeridos por Ronzon y M'Barek $(2018)^{3}$.

Dada la creciente relevancia de la bioeconomía, recientemente se han realizado numerosos esfuerzos para su medición y el cálculo de su impacto (Wesseler \& Braun, 2017; Bracco et al., 2018; Efken et al., 2016; Ronzon \& M'barek, 2018; M'barek et al., 2014). La mayoría de los estudios se basan en análisis de variables macroeconómicas como la generación de empleo, el valor añadido de los sectores bioeconómicos o la inversión en biotecnologías y/o biorrecursos. Sin embargo, uno de los principales escollos en estos análisis es la correcta definición de los sectores y subsectores que deben estar englobados dentro de la bioeconomía dado su número y diversidad (Lainez et al., 2019). Así, la falta de claridad en la definición de «biorrecursos» y la imprecisa delimitación de los «sectores bioeconómicos» complican los análisis comparativos tanto entre países, como dentro de cada país (entre regiones o sectores). A esto se suma la crítica generalizada de que las mediciones de la bioeconomía sobreestiman su relevancia ya que importantes sectores como la producción de plásticos, textiles, químicos o energía, que no son totalmente sectores biobasados, se computan en su totalidad en la medición de la bioeconomía (Wydra, 2020). Finalmente, la complejidad analítica se incrementa si además contemplamos la noción de

'Datos para la Unión Europea de los 27 y descontando la contribución de Reino Unido.

2 Última estimación disponible para 2017.

${ }^{3}$ Los diez sectores bioeconómicos considerados son (entre paréntesis se muestra el código NACE-Clasificación Europea de Actividades Económicas): 1. Agricultura (A01). 2. Comida, bebida y tabaco (C10C11-C12). 3. Industria maderera y muebles (C16 y C31). 4. Textiles biobasados (C13-C14-C15). 5. Papel (C17). 6. Industria forestal (A02). 7. Químicos, productos farmacéuticos, plásticos y gomas (C20-C21-C22). 8. Pesca y acuicultura (A03). 9. Electricidad biobasada (D3511). 10. Biocombustibles líquidos: biodiesel (C2059) y bioetanol (C2014). 
circularidad, la convergencia sectorial y la simbiosis industrial que caracterizan al modelo bioeconómico (Sanz-Hernández, Esteban et al., 2019).

El Gráfico 1 muestra que más de la mitad de la contribución de la bioeconomía viene de dos sectores fundamentales como son el sector agrícola y la industria agroalimentaria. Entre estos dos sectores se genera más del $65 \%$ del valor añadido total de la bioeconomía; pero, además, ambos emplean al $78 \%$ de los trabajadores dedicados a sectores bioeconómicos (Gráfico 2). El tercer sector en términos de relevancia es la industria química, farmacéutica y de elaboración de bioplásticos, aunque su importancia está muy lejos del impacto del sector agrícola y la industria agroalimentaria a él asociada. Además, si bien este sector es el tercero más relevante en términos de generación de valor añadido, ocupa la cuarta posición en generación de puestos de empleo, muy por detrás de la industria de la madera y el mueble (cuarta en su aportación al valor añadido y tercera en generación de empleo). Finalmente, un hecho relevante que se desprende de este gráfico es el todavía escaso peso que tienen la generación de bioelectricidad y biocombustibles líquidos. A pesar de la potenciación de estas energías durante los últimos años, estas actividades económicas aportan menos del $2 \%$ del total del valor añadido y solo suponen un $0,5 \%$ del total de empleos en bioeconomía dentro la UE-27.

\section{GRÁFICO 1}

VALOR AÑADIDO EN MILLONES DE EUROS CORRIENTES POR SECTORES BIOECONÓMICOS EN LA UNIÓN EUROPEA(UE-27)

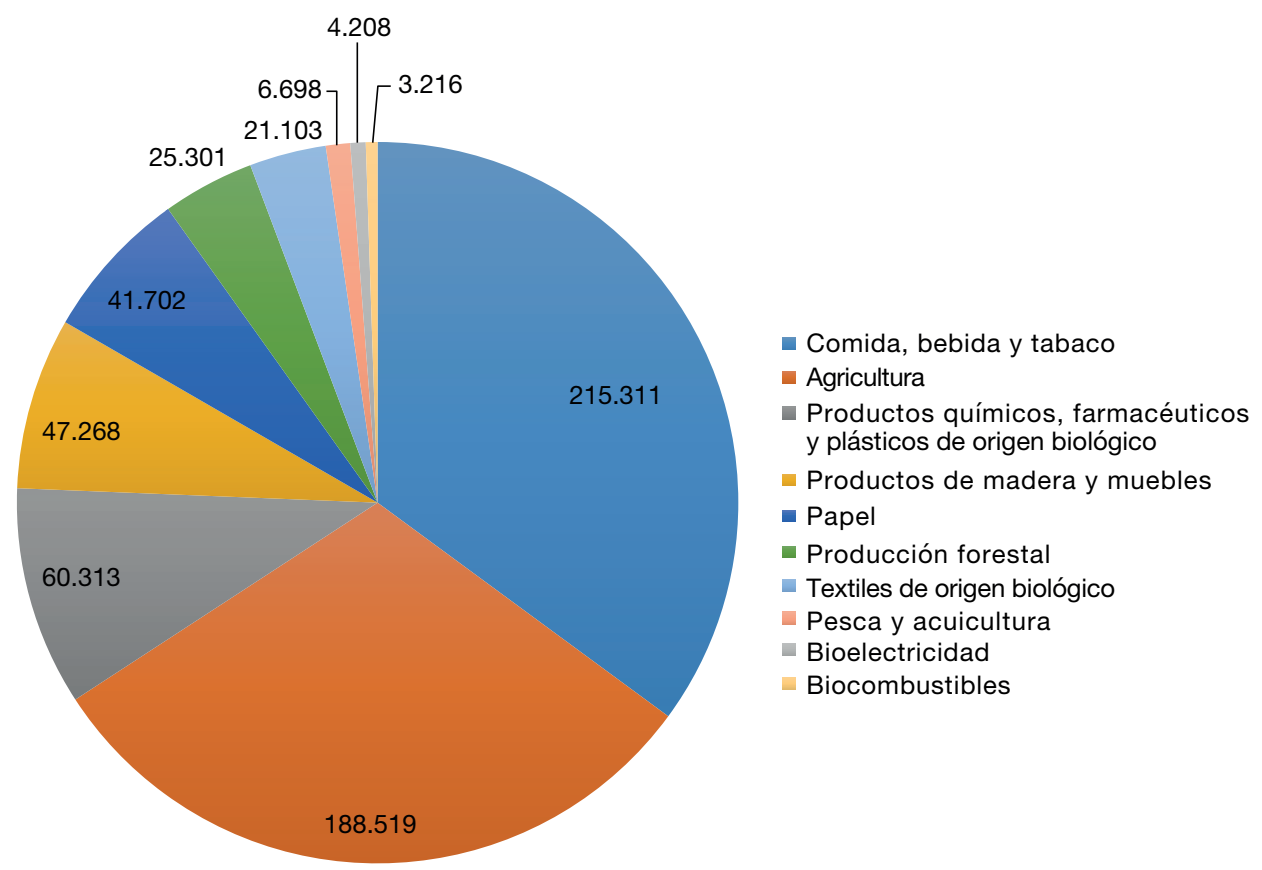

FUENTE: Elaboración propia con datos de 2017 (Ronzon et al., 2020). 


\section{GRÁFICO 2 \\ EMPLEO POR SECTORES BIOECONÓMICOS EN LA UNIÓN EUROPEA}

(UE-27)

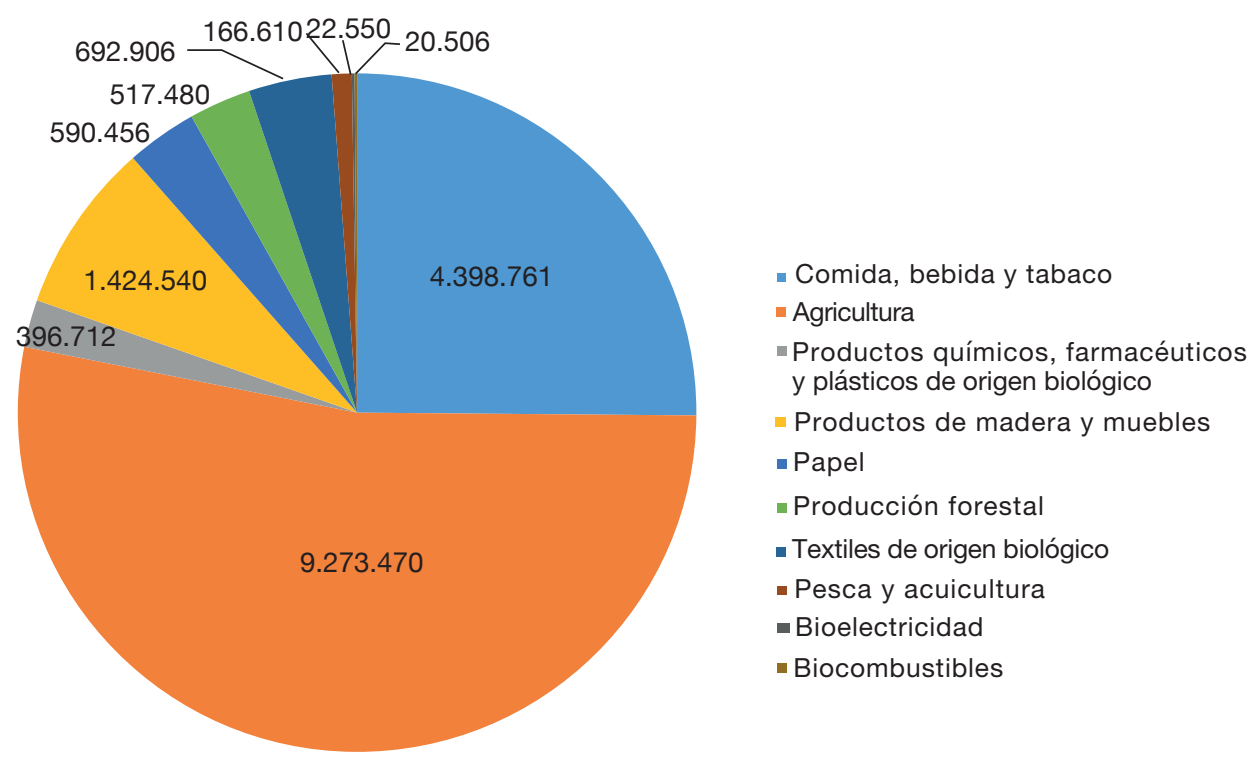

FUENTE: Elaboración propia con datos de 2017 (Ronzon et al., 2020).

A pesar de la relevancia de la bioeconomía en la UE, las actividades bioeconómicas representan un $5 \%$ del total del PIB de la $\mathrm{UE}^{4}$, lo que supone que todavía queda mucho margen de desarrollo y potenciación de estos sectores. Asimismo, se vislumbra estratégico el impulso de sectores con mayor capacidad de generación de valor añadido como son la producción de biocombustibles líquidos, la bioelectricidad o la industria química, farmacéutica y de bioplásticos.

España es uno de los países de la UE que cuenta con su propia estrategia de bioeconomía a nivel nacional (Ministerio de Economía y Competitividad, 2018). La estrategia española de bioeconomía se ha orientado hacia la potenciación de sectores relacionados con el aprovechamiento de los recursos naturales y biológicos y el desarrollo de la biotecnología. Esta estrategia es relevante, sobre todo, por el peso económico de la agricultura, la industria agroalimentaria y las actividades relacionadas con la generación de biomasa en España. La estrategia española de bioeconomía se asienta en:

- El interés político por participar en los nuevos retos globales y en potenciar sistemas económicos sostenibles.

\footnotetext{
${ }^{4}$ Datos de 2017.
} 
- La creciente importancia de la bioeconomía en el sistema científico español (FECYT, 2017).

- La importante contribución de sectores como el agroalimentario o la biomasa en la producción nacional (Lainez et al., 2018).

\section{La bioeconomía en España}

Tres grupos de indicadores principales pueden ser utilizados para medir el impacto y la evolución de los sectores bioeconómicos (Jander et al., 2020): (i) indicadores económicos (empleo, valor añadido, exportaciones, cifra de negocio, creación/ número de empresas, etc.); (ii) indicadores medioambientales (consumo de energía, huella climática, reducción de emisiones, etc.); (iii) indicadores de innovación (inversión pública y privada en $\mathrm{I}+\mathrm{D}$, patentes, publicaciones, desarrollo de nuevos productos, etc.). La dificultad en la delimitación de la bioeconomía y, sobre todo, la falta de información genera que los indicadores económicos sean los más utilizados para medir el impacto y la evolución de la misma (Wydra, 2020).

Para la estimación de la contribución y peso de la bioeconomía en España, se ha adoptado la categorización de recientes estudios que parten de la clasificación realizada por la Comisión Europea (Ronzon et al., 2020; D’Adamo, 2020) y que atiende a los criterios centrales de simplicidad y comparabilidad, lo que nos permite comparar la repercusión y evolución de los distintos sectores bioeconómicos en España y en la UE. Los datos utilizados en este trabajo han sido obtenidos del portal de la Comisión Europea (DataM) que recopila y estima datos económicos sobre agricultura y recursos naturales (Ronzon et al., 2020) ${ }^{5}$. La información disponible en el momento de cierre de este estudio para variables como el valor añadido, cifra de negocio y empleo, contempla el periodo comprendido entre 2008 y 2017. El resto de información (inversión en I+D, inversión en biotecnología, etc.) ha sido obtenida a partir de las estadísticas oficiales del Instituto Nacional de Estadística de España (INE) ${ }^{6}$. Según el Instituto Nacional de Estadística las cifras sobre biotecnología «tienen como objetivo medir el esfuerzo nacional en actividades relacionadas con la biotecnología, de manera que pueda suministrar la información necesaria para la toma adecuada de decisiones en política científico-tecnológica». Estas cifras permiten determinar aquellos sectores que están más relacionados con la bioeconomía y estimar su producción, su actividad y el empleo que se genera en los mismos.

\footnotetext{
${ }^{5}$ https://datam.jrc.ec.europa.eu/datam/public/pages/index.xhtml?rdr=1598371278832. Fecha de consulta de datos en agosto de 2020 .

${ }^{6}$ https://www.ine.es/dyngs/INEbase/es/operacion.htm?c=Estadistica_C\&cid=1254736176808\&menu=ulti Datos\&idp $=1254735576669$.

https://www.ine.es/dyngs/INEbase/es/operacion.htm?c=Estadistica_C\&cid=1254736176808\&idp=12547355

$76669 \&$ menu $=$ ultiDatos. Fecha de consulta de datos en agosto de 2020 .
} 


\subsection{El impacto de la bioeconomía en España}

La bioeconomía aporta en España un total de 65 billones, lo que supone un $11 \%$ del valor añadido generado por la Unión Europea (UE-27). En 2017 la bioeconomía en España contribuyó en más de un $6 \%$ al PIB español, lo que supone un punto porcentual más, comparado con la media de la UE. En cuanto al empleo, en 2017, los sectores bioeconómicos ocupaban en España a casi un millón y medio de trabajadores, lo que supone casi un $8 \%$ del total de empleados en España, situando al país en la quinta posición atendiendo al empleo dedicado en estos sectores. Además, es importante señalar que cada trabajador español en sectores asociados a la bioeconomía genera un valor añadido de 46.000 euros, lo que supone un $31 \%$ más que la contribución media por trabajador en la UE (35.000 euros por trabajador).

Si analizamos la contribución por sectores bioeconómicos en España (Gráfico 3) se observa un patrón muy similar a lo que ocurre en la UE (Gráfico 1). En el caso de España, el primer sector por relevancia es la agricultura seguido de la industria agroalimentaria (comida, bebida y tabaco). Esto evidencia cómo la bioeconomía en España está más especializada en sectores que generan un menor valor añadido (más

\section{GRÁFICO 3}

\section{VALOR AÑADIDO EN MILLONES DE EUROS POR SECTORES} BIOECONÓMICOS EN ESPAÑA

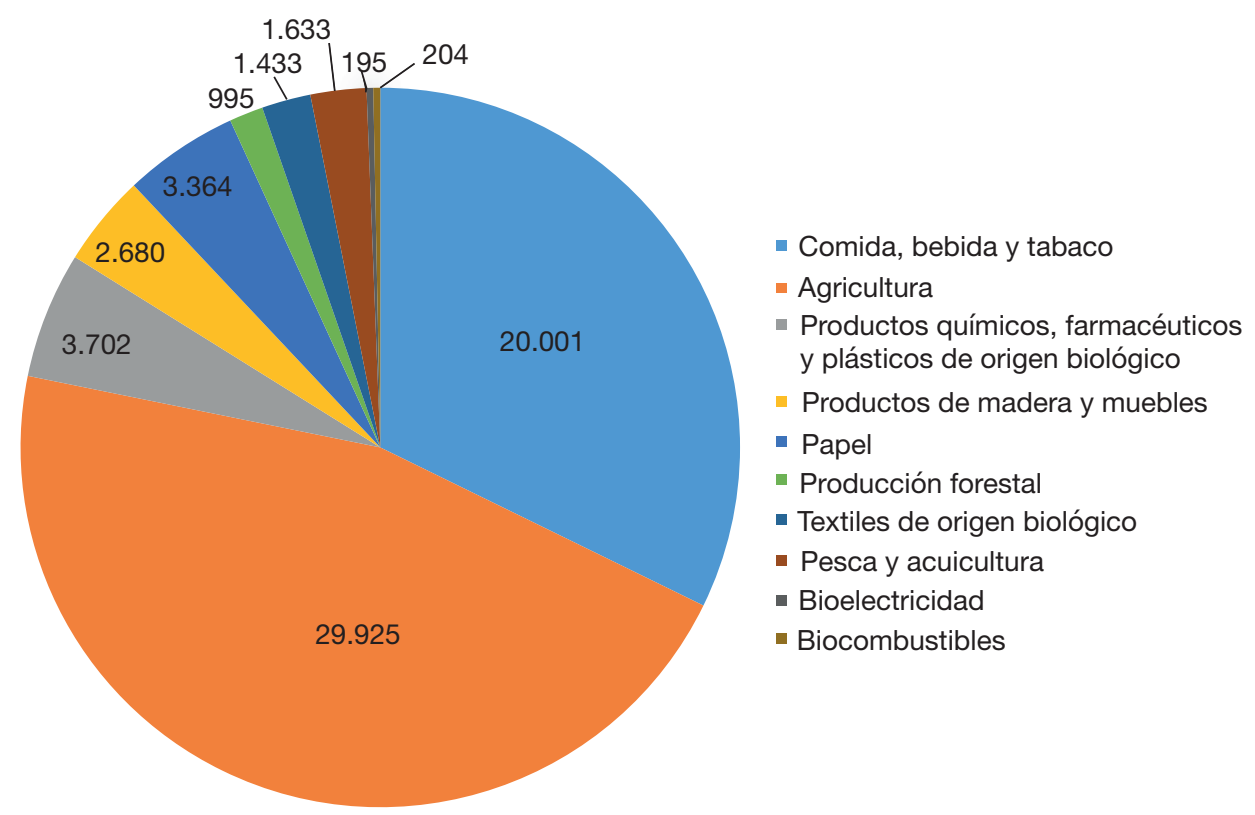

FUENTE: Elaboración propia con datos de 2017 (Ronzon et al., 2020). 
bajo en la agricultura que en la industria agroalimentaria). Asimismo, el peso de la industria química, farmacéutica y la generación de bioplásticos es proporcionalmente inferior en España comparado con la UE de los 27. Mientras que esta industria aporta casi un $10 \%$ del valor añadido para el conjunto de la UE-27, en el caso de España supone solamente un 5,6\%. Lo mismo ocurre en el caso de la generación de bioelectricidad y biocombustibles líquidos, que en España representan un 0,7\% del valor añadido en bioeconomía, frente al 1,2\% europeo.

La evolución del valor añadido por sectores bioeconómicos desde 2008 hasta 2017 (Cuadro 1) muestra que, tanto para la UE-27 como para España, la tasa de variación es negativa durante los años próximos a la crisis económica de 2008 . No obstante, en el caso español la evolución es peor que la media europea. Desde 2008 se observa una importante caída del valor añadido generado por sectores bioeconómicos, superior para el caso español. En el caso de España solo se alcanza el nivel de 2008 en el año 2016, mientras que en la UE-27 esta cifra se recupera e incluso se supera en 2011. Además, si bien España presenta datos positivos para 2016 y 2017 , la tasa de crecimiento de estos años está notablemente alejada de las tasas de crecimiento de la bioeconomía en la UE. De nuevo, estos datos evidencian que en España hace falta un mayor esfuerzo en estos sectores para poder alcanzar el ritmo de crecimiento europeo.

\section{CUADRO 1}

TASA DE VARIACIÓN DEL VALOR AÑADIDO DE LA BIOECONOMÍA

\begin{tabular}{|l|r|r|r|r|r|r|r|r|r|}
\cline { 2 - 9 } \multicolumn{1}{c|}{} & $\mathbf{2 0 0 9}$ & $\mathbf{2 0 1 0}$ & $\mathbf{2 0 1 1}$ & $\mathbf{2 0 1 2}$ & $\mathbf{2 0 1 3}$ & $\mathbf{2 0 1 4}$ & $\mathbf{2 0 1 5}$ & $\mathbf{2 0 1 6}$ & $\mathbf{2 0 1 7}$ \\
\hline España & $-8,9$ & $-4,4$ & $-6,0$ & $-9,6$ & $-7,5$ & $-7,1$ & $-1,6$ & 2,7 & 6,7 \\
\hline UE-27 & $-7,2$ & $-0,1$ & 4,0 & 2,9 & 4,8 & 6,9 & 8,7 & 12,5 & 20,6 \\
\hline
\end{tabular}

FUENTE: Elaboración propia a partir de datos de Rozon et al. (2020)

\subsection{La generación de valor añadido y volumen de negocio de los sectores bioeconómicos}

Los datos, de valor añadido y de volumen de negocios, muestran cómo los sectores relacionados con recursos biológicos están ganando en importancia en la economía española (Gráfico 4). Si bien se parte de una tendencia de notable caída en la generación de valor añadido de los sectores englobados dentro de la bioeconomía tras 2008, desde 2012 los datos evidencian una recuperación que se ve afianzada definitivamente en 2016 y 2017 . En el caso de la cifra de negocios, España ha pasado de 198 millones de euros en 2008 a 219 millones de euros en 2017, con un crecimiento sostenido desde 2015. Para el caso de la cifra de negocio, los datos muestran una evolución más estable que para el valor añadido. Es importante señalar que el mayor porcentaje de cifra de negocios se da en el sector 


\section{GRÁFICO 4}

EVOLUCIÓN DEL VALOR AÑADIDO Y CIFRA DE NEGOCIOS

DE LA BIOECONOMÍA EN ESPAÑA

(En millones de euros corrientes)

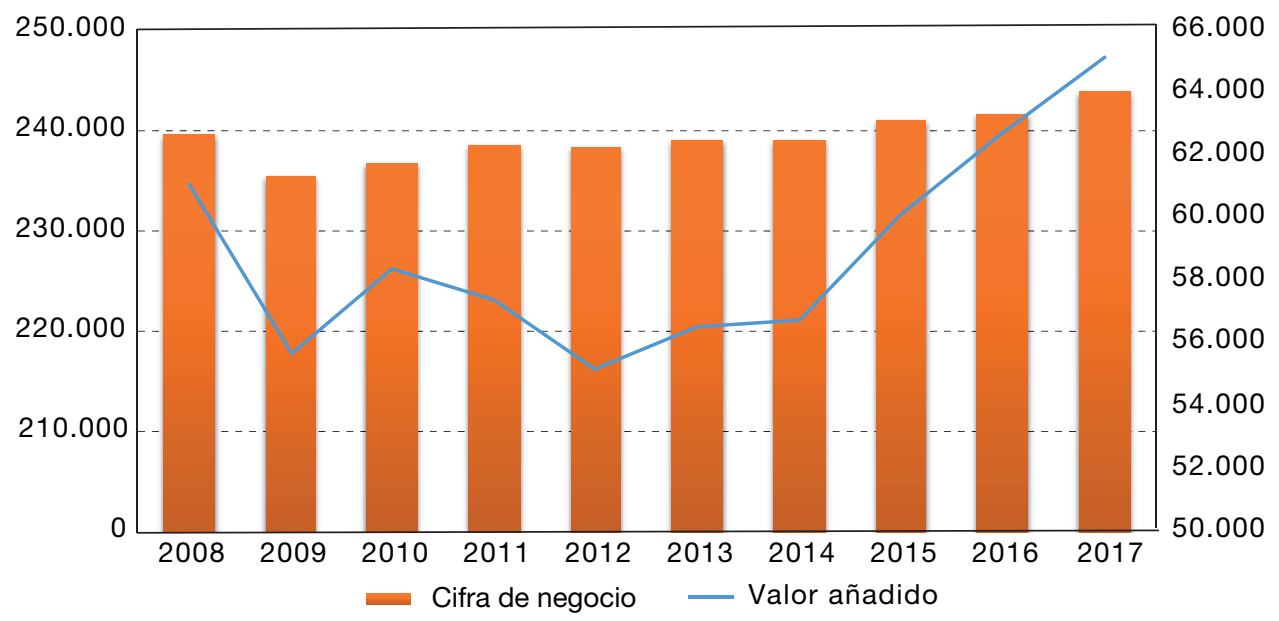

FUENTE: Elaboración propia con datos de Ronzon et al. (2020).

de la industria agroalimentaria seguido de la industria del papel y por último de la industria química, farmacéutica y de bioplásticos. Estos tres sectores bioeconómicos presentan crecimientos sostenidos a lo largo de todo el periodo (Ronzon et al., 2020).

Si se profundiza en la evolución comparativa en los sectores bioeconómicos, a lo largo de la última década, se observan notables diferencias entre el caso español y la UE-27. Las tasas de variación del valor añadido entre sectores bioeconómicos en los contextos español y europeo se recogen en el Cuadro 2. Los resultados muestran una evolución dispar y más negativa para el caso español, ya que mientras que en la UE solo un sector (producción de textiles de origen biológico) disminuye su contribución al valor añadido, en el caso español son cuatro los sectores bioeconómicos que han reducido su aportación al conjunto del valor añadido. Además, los resultados indican que, salvo para los sectores de agricultura y pesca y acuicultura que experimentan crecimientos muy por encima de la media de la UE, el resto de sectores presenta una peor evolución. Son relevantes también las fuertes caídas en sectores como la producción de textiles de origen biológico y en la producción de madera y muebles. Otro dato relevante es el hecho de que España está perdiendo potencial (combustibles líquidos biológicos), o bien, muestra menores crecimientos comparados con la UE (productos químicos, farmacéuticos y plásticos o bioelectricidad), en sectores que son los que mayor margen de beneficios están reportando. 


\section{CUADRO 2}

TASA DE VARIACIÓN DEL VALOR AÑADIDO POR SECTORES BIOECONÓMICOS EN ESPAÑA Y EN LA UE-27 ENTRE 2008 Y 2017

\begin{tabular}{|c|c|c|c|}
\hline Sectores & Subsectores & $\begin{array}{c}\text { Tasa de variación } \\
\text { en España }\end{array}$ & $\begin{array}{c}\text { Tasa de variación } \\
\text { en UE-27 }\end{array}$ \\
\hline \multicolumn{2}{|l|}{ Agricultura } & 25,6 & 18,8 \\
\hline \multicolumn{2}{|l|}{ Producción forestal } & $-32,9$ & 20,6 \\
\hline \multicolumn{2}{|l|}{ Pesca y acuicultura } & 68,3 & 26,7 \\
\hline \multirow{4}{*}{ Comida, bebida y tabaco } & Total & 0,5 & 23,3 \\
\hline & Comida & 3,3 & 21,9 \\
\hline & Bebida & $-5,9$ & 33,6 \\
\hline & Tabaco & $-32,7$ & 2,5 \\
\hline \multirow{4}{*}{ Textiles de origen biológico } & Total & $-32,3$ & $-3,4$ \\
\hline & Textiles de origen biológico & $-14,4$ & $-0,4$ \\
\hline & $\begin{array}{l}\text { Prendas de vestir origen } \\
\text { biológico }\end{array}$ & $-58,5$ & $-24,9$ \\
\hline & Pieles & $-4,4$ & 29,3 \\
\hline \multirow{3}{*}{$\begin{array}{l}\text { Producción de madera } \\
\text { y muebles }\end{array}$} & Total & $-43,8$ & 1,3 \\
\hline & Productos de madera & $-36,0$ & 1,9 \\
\hline & Muebles & $-56,5$ & 0,2 \\
\hline \multicolumn{2}{|l|}{ Papel } & 1,4 & 18,3 \\
\hline \multirow{4}{*}{$\begin{array}{l}\text { Productos químicos, } \\
\text { farmacéuticos y plásticos }\end{array}$} & Total & 13,6 & 43,3 \\
\hline & Químicos & 21,4 & 62,1 \\
\hline & Farmacéuticos & 14,0 & 40,0 \\
\hline & Gomas y plásticos & 0,4 & 43,2 \\
\hline \multirow{3}{*}{$\begin{array}{l}\text { Combustibles líquidos } \\
\text { biológicos }\end{array}$} & Total & $-7,9$ & 37,7 \\
\hline & Bioetanol & 4,2 & 133,2 \\
\hline & Biodiesel & $-12,7$ & $-3,5$ \\
\hline \multicolumn{2}{|l|}{ Bioelectricidad } & 100,0 & 140,0 \\
\hline \multicolumn{2}{|l|}{ TOTAL VALOR AÑADIDO } & 6,7 & 20,6 \\
\hline
\end{tabular}

FUENTE: Elaboración propia a partir de datos de Rozon et al. (2020).

\subsection{El empleo de los sectores bioeconómicos}

Los datos del empleo en bioeconomía para España muestran una tendencia decreciente hasta 2014 y una leve recuperación del empleo en sectores bioeconómicos desde 2015 (Gráfico 5). A diferencia de lo que se observaba en el caso del valor añadido, es importante señalar que, a pesar del aumento del empleo en estos sectores 


\section{GRÁFICO 5 \\ EVOLUCIÓN DEL NÚMERO DE EMPLEADOS EN SECTORES RELACIONADOS CON LA BIOECONOMÍA EN ESPAÑA (En millones de empleados)}

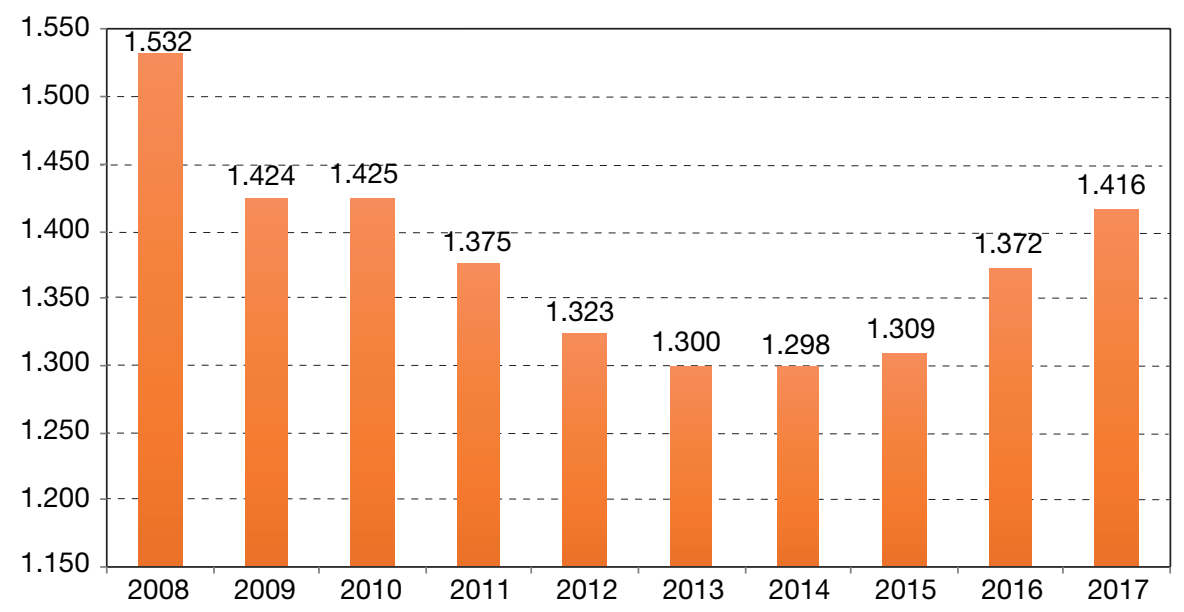

FUENTE: Elaboración propia a partir de datos de Ronzon et al. (2020).

durante los últimos años, no se ha conseguido alcanzar el nivel de 2008. Parte de los años que se analizan coinciden con el periodo de la crisis financiera de 2008, en donde España experimentó un fuerte crecimiento de la tasa de paro, lo que en parte podría explicar las cifras reflejadas. Frente a ello, los datos del valor añadido confirmaban que en 2016 se sobrepasan los niveles de 2008 y existía una tendencia hacia la recuperación desde 2012. Una explicación a estas desiguales tendencias en los resultados es que España está creciendo en sectores que no potencian la creación de empleo y ha reducido potencial en aquellos sectores que requerían mayores niveles de ocupación.

Un análisis pormenorizado de la evolución del valor añadido y empleo por sectores puede observarse en el Gráfico 6. En este gráfico se refleja cómo los sectores que muestran crecimiento en el caso español, o bien potencian el empleo en una proporción mucho menor o bien destruyen empleo (por ejemplo, en el caso del sector de pesca y acuicultura). A esto se suma que los sectores que han disminuido su contribución al valor añadido, han destruido mayores niveles de empleo comparado con la contracción en el valor añadido (salvo para la producción forestal). Los resultados sobre el empleo ponen de manifiesto que España tiene todavía mucho trabajo pendiente para potenciar no solo el crecimiento de la bioeconomía sino también para el fortalecimiento de sectores que arrastran mayores niveles de empleabilidad. No obstante, es importante indicar que en el caso de la UE-27 se observa también una reducción de empleo, incluso mayor que la española, en los sectores relacionados con la bioeconomía. 


\section{GRÁFICO 6}

\section{TASA DE VARIACIÓN DEL VALOR AÑADIDO Y DEL EMPLEO EN BIOECONOMÍA EN ESPAÑ̃ \\ (En \%)}

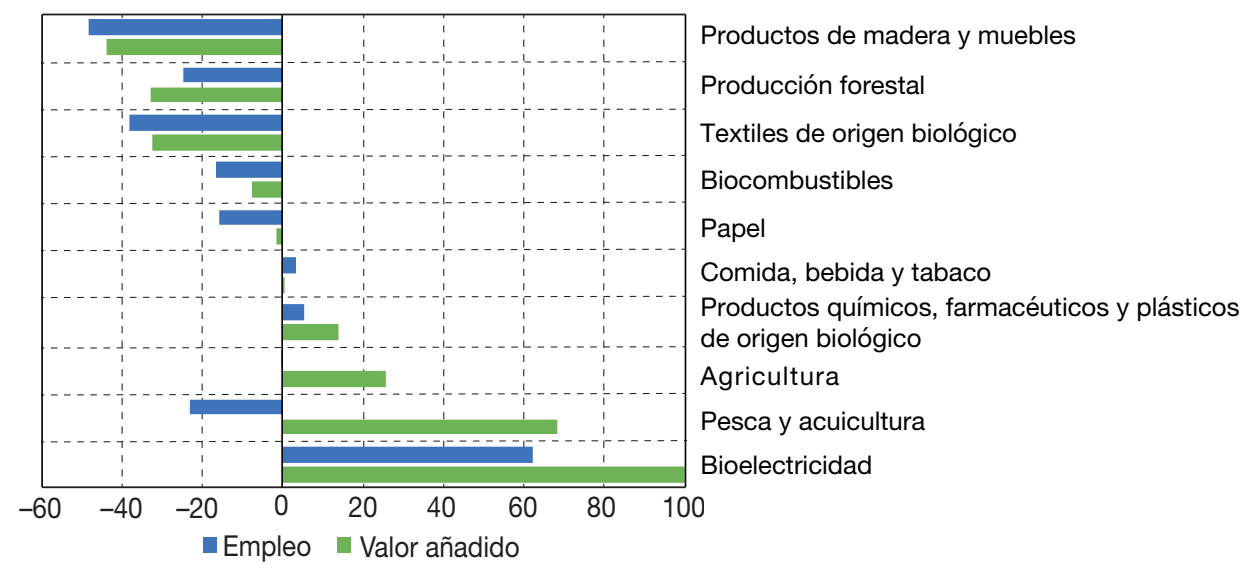

FUENTE: Elaboración propia a partir de datos de Ronzon et al. (2020).

\subsection{Una comparación con algunos países de la $U E$}

España es, actualmente, la cuarta potencia de la UE-27 en términos de PIB y la quinta si se tiene en cuenta a Reino Unido. La comparación de España con las principales economías de la UE-27 evidencia que, a pesar de que España está mejorando y creciendo en los sectores bioeconómicos, todavía queda mucho trabajo pendiente.

En el Gráfico 7 se realiza una comparación de la evolución de la generación de valor añadido de la bioeconomía, entre 2008 y 2017, en las principales potencias europeas: Alemania, Francia, Italia, Reino Unido y España. Los resultados revelan que España no solo está en la cola en cuanto a la generación de valor añadido, sino que además la tasa de crecimiento de los sectores bioeconómicos (en términos de generación de valor añadido) es muy inferior al crecimiento que muestran el resto de grandes potencias, exceptuando Reino Unido.

Alemania, Francia e Italia presentan un fuerte desarrollo de la bioeconomía y una gran capacidad de generación de valor añadido. En 2008 mostraban valores de generación de valor añadido cercanas (sobre todo, Francia e Italia) e incluso por encima de los 80 billones de euros (Alemania). En 2017, los datos evidencian el gran incremento que se ha producido en los distintos sectores bioeconómicos. En el caso de Alemania y Francia, durante este periodo de tiempo, el valor añadido generado por la bioeconomía se ha incrementado en unos 20 billones de euros, mientras que en el caso de la economía italiana el incremento ha sido de 11 billones de euros. Por otro lado, están España y Reino Unido que presentan una evolución muy similar, con niveles de generación de valor añadido alrededor de los 60 billones de euros en 2008 y muy lejos de los cerca de 80 billones que registran el resto de grandes potencias. La 


\section{GRÁFICO 7 \\ EVOLUCIÓN DEL VALOR AÑADIDO}

(En miles de millones corrientes)

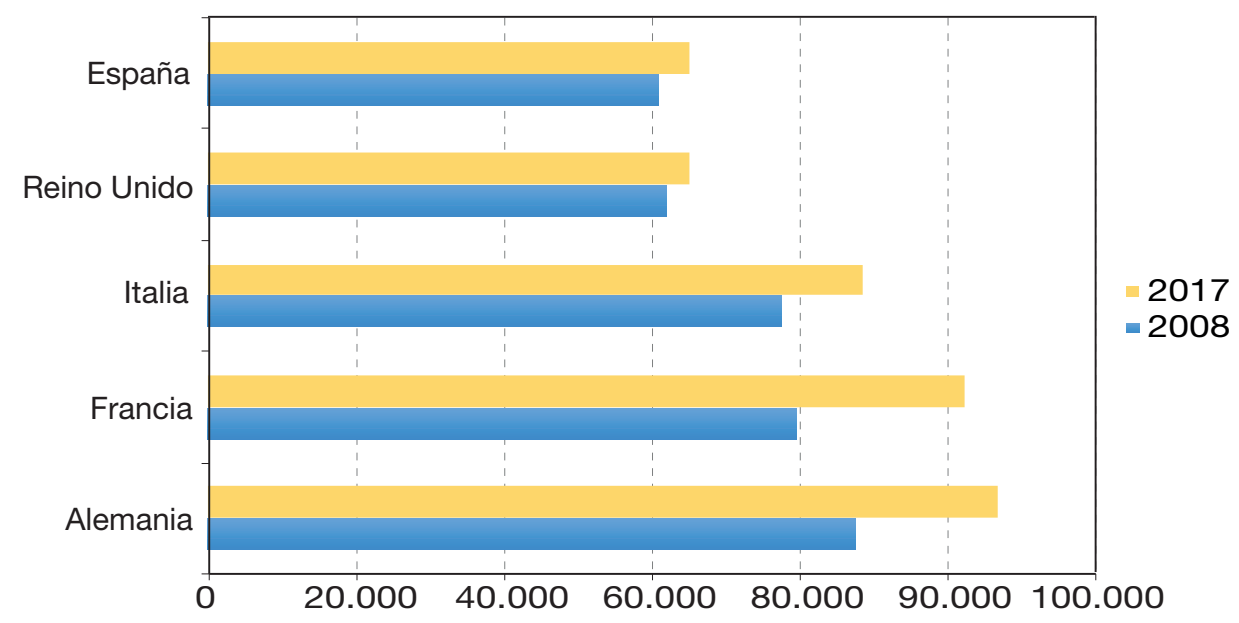

FUENTE: Elaboración propia (Ronzon et al., 2017).

análoga evolución del Reino Unido y España indica que en un periodo de ocho años estas economías solo han conseguido aumentar el valor añadido en bioeconomía en aproximadamente 3 billones de euros.

Estas cifras reflejan que España está todavía por detrás de los principales países europeos en el desarrollo de la bioeconomía. Si bien los datos manifiestan un crecimiento en la contribución de la bioeconomía, ni el valor ni su evolución están al nivel de las grandes potencias. No obstante, a pesar del menor desarrollo español en sectores bioeconómicos, si analizamos la contribución de la bioeconomía al valor añadido en los diferentes países, en España la bioeconomía tiene una mayor relevancia ya que supone un $6,2 \%$, mientras que esta cifra está en torno al 3,5\% en Alemania, al 5\% en Francia y al 5,7\% en Italia.

Otro resultado relevante es la contribución de los distintos sectores al valor añadido. En este sentido, en el caso de España, en 2017, la agricultura aporta un $46 \%$ del valor añadido total de bioeconomía, seguido de la industria agroalimentaria con un $32 \%$. Sin embargo, la agricultura supone alrededor del $20 \%$ en el caso de Alemania y Reino Unido donde la industria agroalimentaria (comida, bebida y tabaco) aporta al valor añadido de la bioeconomía un $41 \%$ y un $53 \%$, respectivamente. En el caso de Francia, que cuenta con un sector agrario importante, la contribución de la agricultura al valor añadido de la bioeconomía es del $31 \%$, mientras que la industria agroalimentaria contribuye en un $45 \%$. Finalmente, el caso de Italia es muy similar al español con una contribución de la agricultura del $36 \%$ y de la industria agroalimentaria del $26 \%$ del valor añadido total generado por la bioeconomía. 
No obstante, en el caso de la economía italiana es importante resaltar la importancia de la contribución de sectores como la producción de madera (11\%) y la industria textil de origen biológico (11\%).

Por su parte, la comparación del empleo muestra resultados dispares entre los países seleccionados. En el Gráfico 8 se observa que el empleo en sectores relacionados con la bioeconomía aumenta en Reino Unido y en Francia, aunque en este segundo caso lo hace en un porcentaje muy bajo. Por el contrario, en España, Italia y Alemania se produce una destrucción de empleo en estos sectores. Si bien, la caída de empleados más notable es la que se da para el caso de la economía italiana, en España también se percibe una disminución relevante.

Finalmente, para completar el análisis realizado, incorporamos los resultados de un reciente estudio de D'Adamo et al. (2020) donde se propone un indicador denominado Socioeconomic indicator for the bioeconomy (SEIB). Este indicador mide el desempeño socioeconómico de los sectores de la bioeconomía en el contexto europeo, siguiendo las propuestas de O'Brien et al. (2011) y Ronzon y M'Barek (2018). Los resultados de este indicador muestran que los países europeos con mejor desempeño y consecuentemente mejor posicionados para jugar un papel clave en el desarrollo de los sectores de la bioeconomía en los próximos años, son Irlanda, Dinamarca y Países Bajos. El estudio citado coloca a España por encima de la media europea en desempeño bioeconómico, ocupando el séptimo lugar, junto a otras grandes potencias como Francia o Alemania. Además, este estudio confirma los resultados presentados en este trabajo y resalta cómo, en general, España está por detrás de la media europea en desarrollo bioeconómico, salvo por el caso del sector agrario que tiene un peso muy por encima de la media europea.

\section{GRÁFICO 8 \\ EVOLUCIÓN DEL EMPLEO}

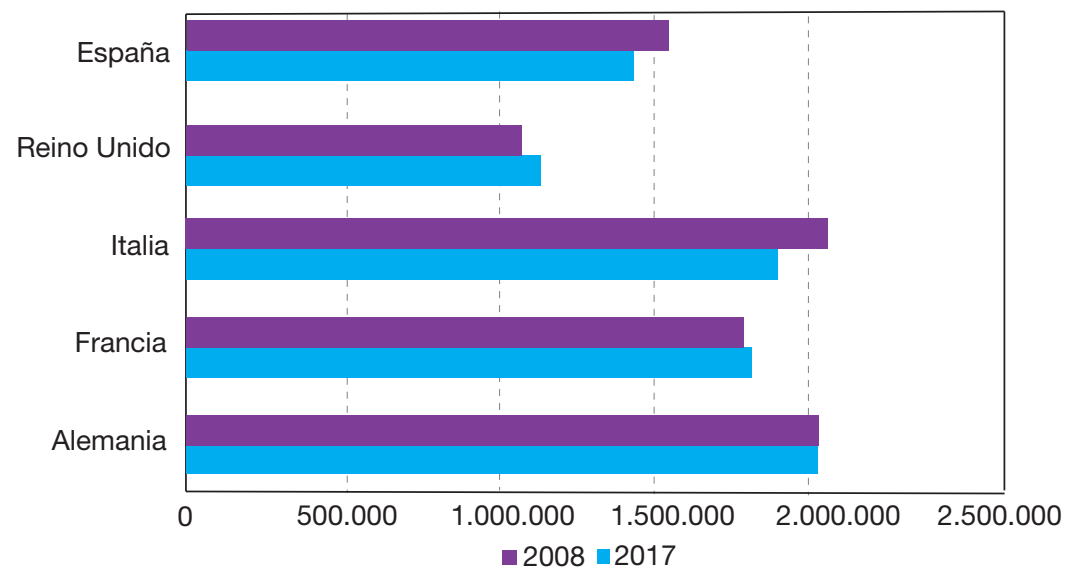

FUENTE: Elaboración propia (Ronzon et al., 2017). 
En el caso español, la excesiva especialización y dependencia de sectores como el agrícola, con menor capacidad de crecimiento y de generación de valor añadido, es uno de los mayores hándicaps para el crecimiento de la bioeconomía, pese a estar en el grupo de los países europeos considerados mejor posicionados para el desempeño bioeconómico: Irlanda, Dinamarca, Países Bajos, Austria, Portugal, Rumanía, España y Grecia (D’Adamo et al, 2020). España mejoraría su posición en relación con el resto de Europa si incrementase la actividad en microsectores más competitivos vinculados con la fabricación industrial y la bioenergía. Sin embargo, la pérdida de importancia y/o bajo crecimiento de estos sectores ha llevado a España a colocarse en el grupo de países rezagados en este ámbito específico junto con Países Bajos, Estonia, Polonia, Italia, República Checa, Hungría, Letonia, Grecia, Suecia, Rumania, Chipre, Eslovenia, Luxemburgo, Eslovaquia y Malta.

\section{El gasto en actividades de I+D en bioeconomía}

Además de la contribución de los sectores bioeconómicos al empleo y al valor añadido otra serie de variables son relevantes para tener una visión general de la evolución y estado de la bioeconomía en España. Un indicador destacado es la inversión en investigación, desarrollo e innovación en esta materia ${ }^{7}$.

\subsection{El impulso de la biotecnología en España}

Si analizamos el gasto en actividades de I+D en biotecnología en España advertimos una trayectoria creciente desde 2014 , fecha en la que se destinaba un total de 1.300 millones de euros al desarrollo de biotecnología. Esta cifra se elevó hasta los casi 1.800 millones de euros en 2018. El fuerte impulso de estos años en biotecnología demuestra el interés creciente de la economía española en potenciar este tipo de sectores. Si analizamos los datos del Gráfico 9, que representa la inversión en I+D en biotecnología, se observa una trayectoria ascendente hasta 2010. El periodo entre 2011 y 2014 se caracteriza por una disminución del gasto que puede deberse a la contracción económica que sufrió España como consecuencia de la crisis financiera de 2007. Pero desde 2014 los datos muestran un sostenido crecimiento del gasto en biotecnología.

Si se presta atención al origen de estos gastos, las cifras indican que en 2008 el mayor porcentaje proviene de la inversión de las Administraciones públicas (40\% Administraciones públicas, $23 \%$ educación superior y $37 \%$ sector privado), mientras que en 2018 se ha producido un cambio en este patrón, siendo las empresas las primeras en cuanto a contribución (con un $40 \%$ del gasto en biotecnología),

\footnotetext{
7 Información obtenida del INE. https://www.ine.es/dyngs/INEbase/es/operacion.htm?c=Estadistica C\&cid $=1254736176808 \& i d p=1254735576669 \&$ menu=ultiDatos. Fecha de consulta agosto 2020 .
} 


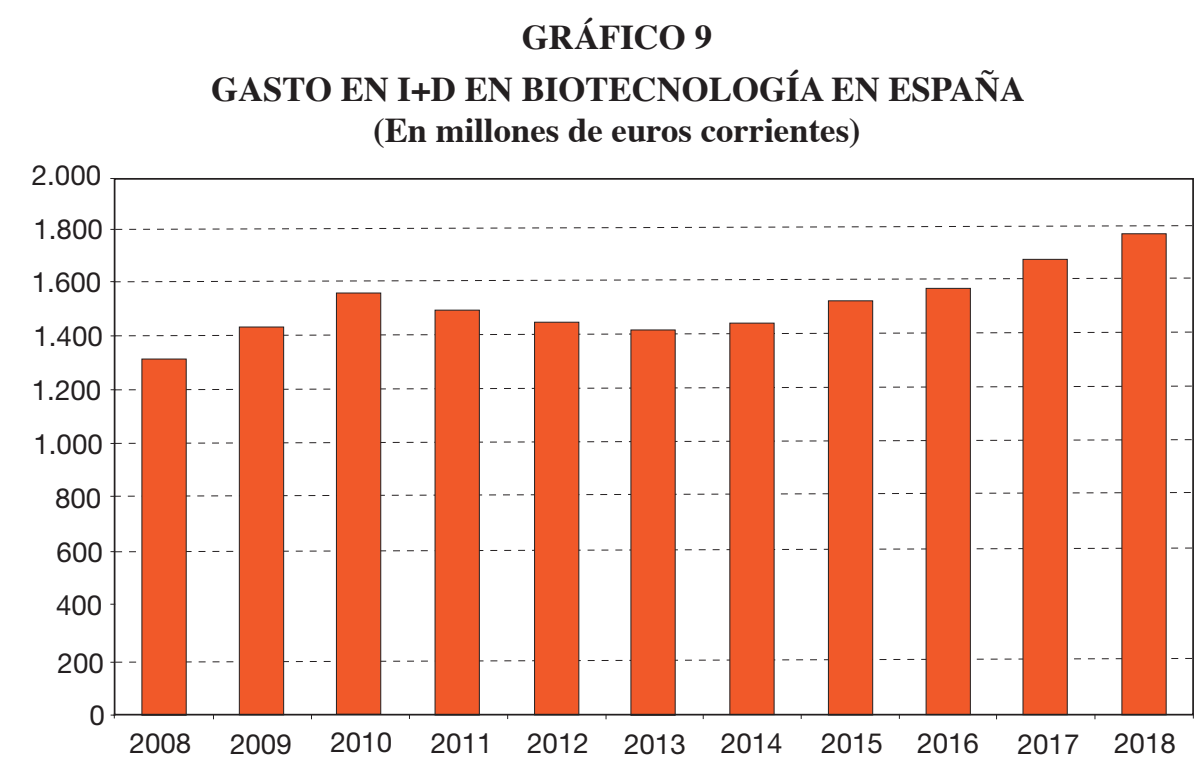

FUENTE: Elaboración propia con datos del INE (Estadísticas sobre el uso de biotecnología).

seguidas de las Administraciones públicas con un porcentaje del $36 \%$. Esto evidencia el esfuerzo tanto público como privado en destinar inversiones a desarrollos en biotecnología; además, pone de manifiesto la centralidad y liderazgo empresarial actual en el sector durante los últimos años (Sanz-Hernández, Sanagustín-Fons et al., 2019). No obstante, es importante señalar que, a pesar del crecimiento en el gasto por parte del sector privado, la mayor parte de la financiación proviene todavía del conjunto de Administraciones públicas y educación superior, que en su mayoría se sufraga a través de fondos públicos. Finalmente, otro hecho relevante son los sectores a los que están dedicados esos gastos en I+D en biotecnología. El mayor porcentaje del gasto se concentra en el sector servicios que atrae al $68 \%$ de inversión en biotecnología, seguido por el sector industrial con un $28 \%$ y, finalmente, el sector primario con un $4 \%$.

Otro análisis sugerente para entender la evolución de la inversión en biotecnología en España es el estudio de la distribución espacial de estos gastos. El Gráfico 10, que muestra el porcentaje de gasto en I+D en biotecnología por CC. AA., refleja la existencia de grandes diferencias y la heterogeneidad en la inversión en biotecnologías entre las distintas regiones españolas.

Los datos señalan una alta concentración territorial de la inversión. En general, la suma de dos comunidades autónomas, la Comunidad de Madrid y Cataluña, engloba casi el $60 \%$ del gasto en biotecnologías. Con una diferencia notable les siguen Andalucía $(11,9 \%)$, Comunitat Valenciana $(8,1 \%)$ y País Vasco $(5,6 \%)$. El resto de CC. AA. muestran un gasto muy bajo o inapreciable. Esto evidencia la dispar repercusión de la bioeconomía en las distintas CC. AA. pese a la abundancia de recursos 


\section{GRÁFICO 10}

DISTRIBUCIÓN DEL GASTO EN I+D EN BIOTECNOLOGÍAS POR CC. AA.

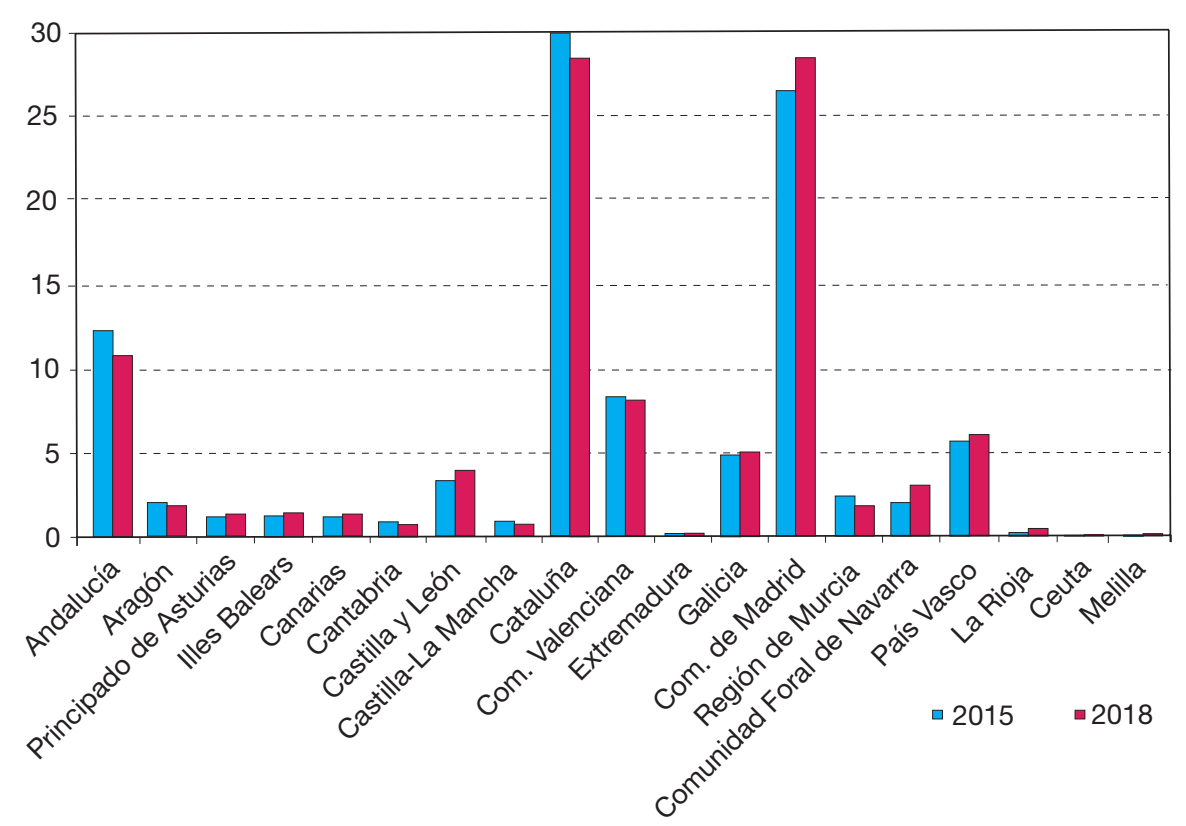

FUENTE: Elaboración propia con datos del INE (Estadísticas sobre el uso de biotecnología).

naturales y biológicos. Además, pone de manifiesto las diferencias en las políticas de gasto público entre los distintos gobiernos regionales y su desigual interés por la bioeconomía. Por ejemplo, si comparamos la inversión entre 2015 y 2018, se observa que Cataluña ha reducido su gasto en biotecnología y en 2018 pasa a ser la segunda CC. AA. por detrás de la Comunidad de Madrid. Por otro lado, resalta también la disminución de gasto en el caso de Andalucía o la estabilización del mismo en la Comunitat Valenciana. En general, los datos reflejan que siete CC. AA. (Illes Balears, Canarias, Castilla y León, Galicia, Comunidad de Madrid, Comunidad Foral de Navarra y País Vasco) han aumentado su gasto en biotecnología, mientras que el resto lo han reducido o lo han mantenido estable.

\subsection{Inversión y producción científica en bioeconomía}

El programa de financiación de la UE «Horizonte 2020» destinado a la financiación de proyectos de investigación e innovación ha considerado a la bioeconomía como una de sus líneas preferentes. Asimismo, son numerosos los programas europeos que han venido destinando parte de su financiación al desarrollo, potenciación e investigación en materia de bioeconomía. Un ejemplo son los Fondos Estructurales y de Inversión Europeos, que con un presupuesto de 454 billones de euros para el 
periodo 2014-2020, han centrado sus líneas maestras en temas tales como la agricultura y la pesca, el desarrollo rural y la eficiencia energética y la sostenibilidad. El Fondo Europeo para Inversiones Estratégicas, dotado con 315 billones de euros para el periodo 2014-2020, también ha proporcionado financiación para el desarrollo de proyectos sobre bioeconomía. Finalmente, es reseñable la financiación (3,7 billones de euros) dedicada a promover la cooperación y participación entre Administraciones públicas y empresas dedicadas a la biotecnología. La fuerte inversión de la UE en materia de bioeconomía y biotecnología demuestra la notable importancia que tiene la bioeconomía en el contexto europeo e incentiva las expectativas políticas y económicas que se vienen generando en torno al rol futuro que puede desempeñar (Ministerio de Economía y Competitividad, 2018).

En el caso español, tal y como se ha presentado en la subsección anterior, la mayor inversión en bioeconomía, representada por el gasto en biotecnología, viene de fondos públicos (Administraciones públicas y educación superior). En 2013, fueron apoyados un total de 778 proyectos en bioeconomía con una financiación total de 124 millones de euros en concepto de inversión directa. A esto se suma la financiación directa vinculada a la Estrategia Española de Bioeconomía que establece una financiación de más de 2.200 millones de euros para la financiación directa de sectores bioeconómicos durante el periodo comprendido entre 2016 y 2020 (Ministerio de Economía y Competitividad, 2017).

Finalmente, en cuanto a la producción científica, España es uno de los países a nivel mundial con mayor presencia y relevancia en la investigación en temas de bioeconomía y biotecnología. En el periodo comprendido entre 2009 y 2014, España ocupó el noveno lugar en el mundo en producción científica en bioeconomía, posicionándose en tercer lugar dentro de la Unión Europea, y solo por detrás de Reino Unido y Alemania. La investigación española en bioeconomía supuso un $1 \%$ del total de la investigación nacional, lo que equivale a un $28 \%$ más que la actividad mundial en esta materia (FECYT, 2017). Además, el Plan Estatal de Investigación Científica y Técnica y de Innovación 2017-2020 (Ministerio de Economía, Industria y Competitividad, 2017) elaborado por el Gobierno de España, al fijar los objetivos y planes fundamentales de la ciencia y la investigación, también ha incluido la bioeconomía como un reto de la sociedad. Este Plan Estatal establece la necesidad de fomentar las actividades de I+D+i en el campo de la bioeconomía para lograr un desarrollo sostenible y avanzar en la implantación de una economía circular.

\section{Discusión y conclusiones}

La creciente preocupación mundial por el medioambiente está facilitando el desarrollo de sistemas de producción más sostenibles y que preserven los recursos naturales. En este contexto la bioeconomía se presenta como una gran oportunidad para potenciar un crecimiento sostenible que garantice el crecimiento de la población especialmente en territorios rurales (Vivien et al., 2019), que asegure la dis- 
ponibilidad de recursos, sobre todo los alimentarios y energéticos, y que mejore la competitividad del crecimiento verde (DeBoer et al., 2019). Sin embargo, no es una propuesta exenta de riesgos (Sanz-Hernández, Esteban et al. 2019), debates (Meyer, 2017) y controversias (Birch et al., 2010).

El proceso hacia un modelo bioeconómico pasa por reajustes en el modelo productivo de los países y por cambios sociotecnológicos y socioecológicos profundos (Melnykovych et al., 2018). Son necesarias transformaciones en la manera de producir, pero también cambios en los patrones de consumo de la sociedad hacia otros estilos de vida (Axon, 2017; 2020) que favorezcan un desarrollo sostenible global sin dejar atrás a nadie, ya sean personas, grupos o territorios, tal y como establece la Agenda 2030 en sus 17 Objetivos de Desarrollo Sostenible -ODS- (Naciones Unidas, 2015). Los gobiernos e instituciones juegan un papel clave para reforzar y estimular la inversión en sectores asociados a la bioeconomía. Además, es necesaria la creación de planes y programas estatales y autonómicos para, en primer lugar, comprometer a todos los grupos afectados -stakeholders- (Reed, 2008), y a los diferentes niveles territoriales (nacional, regional y local), reconociendo la interdependencia entre todos ellos. Finalmente, la implementación del modelo bioeconómico requiere de una concienciación social sobre las implicaciones que conllevan las transiciones de sostenibilidad, definidas estas como «procesos de transformación fundamentales, multidimensionales y a largo plazo a través de los cuales los sistemas sociotécnicos establecidos cambian hacia modos más sostenibles de producción y consumo» (Markard et al., 2012, p. 956). En este sentido, debe reforzarse una educación que desarrolle las competencias transversales y habilidades necesarias para afrontar el reto que supone el modelo bioeconómico, que esté fuertemente asentado en la generación de conocimiento, la innovación tecnológica, social y política y la interdisciplinaridad (Sanz-Hernández, Esteban et al, 2019). La pluralidad de visiones acerca de la bioeconomía hace necesario fomentar la coordinación entre agentes económicos, sociales y políticos (Pfau et al., 2014).

Si bien durante los últimos años se ha dado un impulso institucional fuerte a la bioeconomía, la relevancia de este sector en valor añadido de los países europeos es todavía baja, por ejemplo, en el caso de UE-27 la bioeconomía supone únicamente un $5 \%$ del valor añadido. Son numerosas las barreras, las debilidades y también las opciones políticas, de modo que todavía se requieren muchos esfuerzos para articular las políticas que den respuesta a todas las posibilidades que se abren en los diferentes territorios y actividades económicas y que atiendan a las expectativas que el modelo ha despertado, así como a los intereses de los diferentes actores socioeconómicos.

La actual crisis de la COVID-19, con fuertes colapsos en las economías mundiales, puede suponer un nuevo reto para las políticas medioambientales. Por un lado, esta crisis ha evidenciado la necesidad de proteger el medioambiente y garantizar un desarrollo sostenible que permita minimizar el futuro riesgo de intensificación de pandemias. Por otro lado, los fuertes ajustes económicos que se van a tener que realizar a nivel nacional e internacional pueden minar la actual inversión en la lucha contra el cambio climático. En este sentido, un reto fundamental que van a tener las 
economías y las sociedades a corto plazo es el de seguir manteniendo niveles notables de financiación para la lucha contra el cambio climático y la potenciación de un crecimiento sostenible. La crisis también ha puesto de manifiesto la gran capacidad de los países y de los grupos sociales para luchar en situaciones de emergencia, desplegando respuestas innovadoras que se asientan en la cocreación de valor y en la innovación social.

En el caso de España, la relevancia de la bioeconomía es indudable debido, sobre todo, al fuerte peso que tienen sectores como el agrícola o la industria agroalimentaria en el conjunto de la economía y el potencial que se reconoce en otros como el forestal (Verkerk et al., 2018; Sanz-Hernández, 2021), la gestión de los residuos agrícolas (Egea et al., 2018), la bioenergía (Paredes-Sanchez et al., 2019) y toda la industria manufacturera. Durante los últimos años, España ha impulsado la inversión y el desarrollo en estos sectores y ha potenciado la creación de normativas y estrategias para permitir y facilitar la transición hacia un sistema productivo que garantice la sostenibilidad de los ecosistemas.

Los datos analizados en este estudio confirman que la bioeconomía en España ha aumentado de una manera sostenible su contribución al PIB desde 2014, con crecimientos notables entre los años 2015-2017. Sin embargo, España está todavía excesivamente especializada en aquellos sectores bioeconómicos que generan un menor valor añadido como la agricultura y ha perdido potencial en sectores clave como la generación de bioenergía o la industria química, farmacéutica, bioplásticos o textiles de origen biológico, que han tenido un crecimiento inapreciable. En este sentido, es fundamental replantear las estrategias de bioeoconomía para potenciar aquellos sectores que pueden generar un mayor nivel de valor añadido, así como una mayor empleabilidad. La economía española está lejos del desarrollo de países como Alemania, Francia o incluso Italia, que han sido capaces de potenciar sectores con mayores contribuciones al valor añadido.

El desarrollo de la bioeconomía en España también está reclamando una mayor homogeneidad entre CC. AA. y una mayor armonización del desarrollo territorial, dado que el total de la producción actual en estos sectores está fundamentalmente localizado en la Comunidad de Madrid, Cataluña, Andalucía y Comunitat Valenciana, siendo bajo o muy bajo su desarrollo en el resto de regiones. Son necesarios más estudios que se centren en analizar las barreras existentes para la implementación de la propuesta bioeconómica y revisen los marcos regulatorios y estratégicos con mayor capacidad para impactar positivamente en los territorios y contribuir de manera más efectiva a la reducción de brechas y desigualdades. La Unión Europea va a continuar apoyando la producción de recursos biológicos renovables y su conversión en productos de valor añadido y bioenergía de modo que debe aprovecharse todo el potencial científico español para conocer los recursos inexplorados que podrían ser usados como biocombustibles y bioproductos (Guo \& Song, 2019).

Para concluir, es necesario resaltar que una de las principales limitaciones de este estudio, tal y como se ha apuntado en anteriores secciones, es la dificultad metodológica de la medición de la bioeconomía debido a la inexistencia de datos 
adecuados, fiables y comparables entre niveles territoriales. La información en algunos sectores, como por ejemplo el forestal, es escasa además de poco fiable y se encuentra dispersa (diversidad de fuentes y falta de correspondencia de los datos proporcionados por ellas). Por todo lo anterior, es posible que parte del volumen y peso de las actividades productivas en sectores asociados con la bioeconomía (y consecuentemente el potencial de la misma) se esté minusvalorando. Además, es importante volver a anotar que parte de los datos para la realización del análisis (valor añadido y empleo) solo están disponibles hasta 2017. Como en el caso español es a partir de 2016 cuando se percibe un notable esfuerzo en su desarrollo, cabe esperar que posteriores estudios con nuevas metodologías, indicadores y fuentes muestren cifras con tendencias al alza.

\section{Referencias bibliográficas}

Aquilani, B., Silvestri, C., Ioppolo, G., \& Ruggieri, A. (2018). The challenging transition to bio-economies: Towards a new framework integrating corporate sustainability and value co-creation. Journal of Cleaner Production, 172, 4001-4009. https://doi.org/10.1016/j. jclepro.2017.03.153

Axon, S. (2017). "Keeping the ball rolling": Addressing the enablers of, and barriers to, sustainable lifestyles. Journal of Environmental Psychology, 52, 11-25. https://doi.org/10.1016/j.jenvp.2017.05.002

Axon, S. (2020). The socio-cultural dimensions of community-based sustainability: Implications for transformational change. Journal of Cleaner Production, 266.

https://doi.org/10.1016/j.jclepro.2020.121933

Bell, J., Paula, L., Dodd, T., Németh, S., Nanou, C., Mega, V., \& Campos, P. (2018). EU ambition to build the world's leading bioeconomy - Uncertain times demand innovative and sustainable solutions. (2018). New Biotechnology, 40, Part A, 25-30. https://doi.org/10.1016/j.nbt.2017.06.010

Birch, K., Levidow, L., \& Papaioannou. T. (2010). Sustainable capital? The Neoliberalization of Nature and Knowledge in the European "Knowledge-based Bio-economy". Sustainability, 2, 2898-2918. https://doi.org/10.3390/su2092898

Bracco S, Calicioglu O, Gomez San Juan M, \& Flammini A., (2018). Assessing the Contribution of Bioeconomy to the Total Economy: A Review of National Frameworks. Sustainability, 10(6), 1698. https://doi.org/10.3390/su10061698

Bugge, M., Hansen, T., \& Klitkou, A. (2016). What Is the Bioeconomy? A Review of the Literature. Sustainability, 8, 691-22. https://doi.org/10.3390/su8070691

CE - Comisión Europea. (2012). Innovating for sustainable growth. A bioeconomy for Europe. European Commission.

CE - Comisión Europea. (2018). A sustainable bioeconomy for Europe: Strengthening the connection between economy, society and the environment. European Commission.

D’Amato, D., Droste, N., Allen, B., Kettunen, M., Lähtinen, K., Korhonen, J., Leskinen, P., Matthies, B.D., \& Toppinen, A. (2017). Green, circular, bio economy: A comparative analysis of sustainability avenues. Journal of Cleaner Production, 168, 716-734. https://doi.org/10.1016/j.jclepro.2017.09.053 
D’Adamo, I., Pasquale Marcello Falcone, P.M., \& Morone, P. (2020). A New Socio-economic Indicator to Measure the Performance of Bioeconomy Sectors in Europe. Ecological Economics, 176. https://doi.org/10.1016/j.ecolecon.2020.106724

DeBoer, J., Panwar, R., Kozak, R., \& Cashore, B. (2019). Squaring the circle: refining the competitiveness logic for the circular bioeconomy. Forest policy and economics, 110. https://doi.org/ 10.1016/j.forpol.2019.01.003.

Dresner, S. (2002). The Principles of Sustainability. Earthscan

Efken J., Dirksmeyer W, Kreins P., \& Knecht, M. (2016). Measuring the importance of the bioeconomy in Germany: Concept and illustration. NJAS - Wageningen Journal of Life Sciences 77, 9-17. https://doi.org/10.1016/j.njas.2016.03.008

Egea, F. J., Torrente, R. G., \& Aguilar, A. (2018). An efficient agro-industrial complex in Almería (Spain): Towards an integrated and sustainable bioeconomy model. New Biotechnology, 40, 103-112. https://doi.org/10.1016/j.nbt.2017.06.009

Eurostat, 2008. NACE Rev. 2 Statistical classification of economic activities in the European Community.

https://ec.europa.eu/eurostat/documents/3859598/5902521/KS-RA-07-015-EN.PDF

FECYT - Fundación Española para la Ciencia y la Tecnología. (2017). La producción científica española en el ámbito de la Bioeconomía. 2005-2014.

https://www.fecyt.es/es/publicacion/la-produccion-cientifica-espanola-en-el-ambito-dela-bioeconomia-2005-2014

Georgescu-Roegen, N. (1977). Inequality, Limits and Growth from a Bieconomic Viewpoint. Review of Social Economy 35(3), 361-375. https://doi.org/10.1080/00346767700000041

Gobierno de España (2020). España Circular 2030. Estrategia Española de Economía Circular (consultado el 23 de julio de 2020).

https://www.miteco.gob.es/es/calidad-y-evaluacion-ambiental/temas/economia-circular/ espanacircular2030_def1_tcm30-509532.PDF

Guo, M., \& Song, W. (2019). The growing U.S. bioeconomy: Drivers, development and constraints. New Biotechnology, 49, 48-57. https://doi.org/10.1016/J.NBT.2018.08.005

Ingrao, C., Bacenetti, J, Bezama, A., Blok, V., Goglio, P., Koukios, E.G., Lindner, M., Nemecek, T., Siracusa, V., Zabaniotou, A., \& Huisingh, D. (2018). The potential roles of bio-economy in the transition to equitable, sustainable, post fossil-carbon societies: Findings from this virtual special issue. Journal of Cleaner Production, 204, 417-488. https://doi.org/10.1016/j.jclepro.2018.09.068

Jander, W., Wydra, S., Wackerbauer, J., Grundmann, P., \& Piotrowski, S. (2020). Monitoring Bioeconomy Transitions with Economic-Environmental and Innovation Indicators: Addressing Data Gaps in the Short Term. Sustainablity, 12(11). https://doi.org/10.3390/su12114683.

Jabareen, Y. (2008). A new conceptual framework for sustainable development. Environmental Development and Sustainability, 10(2), 179-192. https://doi.org/10.1007/s10668-006-9058-z

Lainez, M., González, J. M., Aguilar, A., \& Vela. C. (2018). Spanish strategy on bioeconomy: towards a knowledge based sustainable Innovation. New Biotechnology, 40, 87-95. https://doi.org/10.1016/j.nbt.2017.05.006

Markard, J., Raven, R., \& Truffer, B. (2012). Sustainability transitions: an emerging field of research and its prospects. Research Policy, 41(6), 955-967.

https://doi.org/10.1016/j.respol.2012.02.013 
M'barek R, Philippidis, G, Suta, C., Vinyes, C., Caivano, A., Ferrari, E., Ronzon, T., Sanjuan Lopez, A. \& Santini, F. (2014). Observing and analysing the Bioeconomy in the EU - Adapting data and tools to new questions and challenges. Bio-based and Applied Economics, 3(1), 83-91. https://doi.org/10.13128/BAE-14189

McCormick, K. \& Kautto, N. (2013). The Bioeconomy in Europe: An Overview. Sustainability, 5, 2589-2608. https://doi.org/10.3390/su5062589

Melnykovych, M., Nijnik, M., Soloviy, I., Nijnik, A., Sarkki S. \& Bihun, Y. (2018). Social-ecological innovation in remote mountain areas: Adaptive responses of forest-dependent communities to the challenges of a changing world. Science of the Total Environment, 613-614, 894-906. https://doi.org/10.1016/j.scitotenv.2017.07.065

Meyer, R. (2017). Bioeconomy Strategies: Contexts, Visions, Guiding Implementation Principles and Resulting Debates. Sustainability, 9(6), 1031. https://doi.org/10.3390/su9061031

Ministerio de Economía y Competitividad. (2018). Gobierno de España, Secretaria de Estado de Investigación, Desarrollo e Innovación. Estrategia Española de Bioeconomía. Horizonte 2030.

Ministerio de Economía, Industria y Competitividad. (2017). Gobierno de España, Secretaria de Estado de Investigación, Desarrollo e Innovación. Documento reunión de 9 de julio de 2017. Plan Estatal de Investigación Científica y Técnica y de Innovación 2017-2020.

Naciones Unidas (2015). Transforming Our World: the 2030 Agenda for Sustainable Development. https://sdgs.un.org/2030agenda.

O'Brien, K. (2011). Global environmental change II: From adaptation to deliberate transformation. Progress in Human Geography, 36(5), 667-676. https://doi.org/10.1177/0309132511425767

OECD, Organization for Economic Co-operation and Development. Biotechnology for sustainable growth and development. (2004). Science Innovation. https://www.oecd.org/science/emerging-tech/33784888.pdf

OECD, Organization for Economic Co-operation and Development (2009). The Bioeconomy to 2030: designing a policy agenda.

https://www.oecd.org/futures/long-termtechnologicalsocietalchallenges/ thebioeconomyto2030designingapolicyagenda.htm

OECD, Organization for Economic Co-Operation and Development. (2018). Meeting Policy Challenges for a Sustainable Bioeconomy. https://www.oecd.org/publications/policy-challenges-facing-a-sustainablebioeconomy-9789264292345-en.htm

Pfau, S. F., Hagens, J. E., Dankbaar, B., \&. Smits A. J. (2014). Visions of Sustainability in Bioeconomy Research. Sustainability, 6(3), 1222-1249. https://doi.org/10.3390/su6031222

Reed, M. (2008). Stakeholder participation for environmental management: A literature review. Biological Conservation, 141(10), 2417-2431. https://doi.org/10.1016/j.biocon.2008.07.014

Ronzon T., \& M'barek R. (2018). Socioeconomic Indicators to Monitor the EU's Bioeconomy in Transition. Sustainability, 10(6), 1745. https://doi.org/10.3390/su10061745

Ronzon, T., Piotrowski, S., M’barek, R., Carus, M., \& Tamošiūnas, S. (2020). European Commission, Joint Research Centre (JRC). Jobs and wealth in the EU bioeconomy/ $J R C$ - Bioeconomics [Dataset] PID: http://data.europa.eu/89h/7d7d5481-2d02-4b36-8e79-697b04fa4278. 
Sanz-Hernández, A., Esteban, E., \& Garrido, P. (2019). Transition to a bioeconomy: Perspectives from social sciences. Journal of Cleaner Production, 224, 107-119. https://doi.org/10.1016/j.jclepro.2019.03.168.

Sanz-Hernández, A., Sanagustín-Fons, M. V., \& López-Rodríguez, M.E. (2019). A transition to an innovative and inclusive bioeconomy in Aragon, Spain. Environmental Innovation and Societal Transitions, 33, 301-316. https://doi.org/10.1016/j.eist.2019.08.003

Sanz-Hernández, A., Esteban, E., Marco, P., \& Soriano, G. (2020). Forest bioeconomy in the media discourse in Spain. AMBIO: A Journal of the Human Environment, 49, 1897-1911. https://doi.org/10.1007/s13280-020-01390-0

Sanz-Hernández,A. (2021). Privately owned forests and woodlands in Spain: Changing resilience strategies towards a forest-based bioeconomy. Land Use Policy, 100. https://doi.org/10.1016/j.landusepol.2020.104922

Verkerk, P.J., Martinez de Arano, I., \& Palahí, M. (2018). The bio-economy as an opportunity to tackle wildfires in Mediterranean forest ecosystems. Forest Policy and Economics, 86, 1-3. https://doi.org/10.1016/j.forpol.2017.10.016.

Vivien, F.D., Nieddu, M., Befort, N., Debref, R., \& Giampietro, M. (2019). The Hijacking of the Bioeconomy. Ecological Economics, 159, 189-197. https://doi.org/10.1016/j.ecolecon.2019.01.027

Wesseler, J., \& von Braun J. (2017) Measuring the Bioeconomy: Economics and Policies. Annual review of resource economics, 9, 275-298. https://doi.org/10.1146/annurev-resource-100516-053701

Wydra S. (2020). Measuring innovation in the bioeconomy - Conceptual discussion and empirical experiences. Technology in Society, 61.

https://doi.org/10.1016/j.techsoc.2020.101242 